\title{
Apparent diffusion coefficient of vertebral haemangiomas allows differentiation from malignant focal deposits in whole-body diffusion-weighted MRI
}

\author{
Jessica M. Winfield ${ }^{1,2}$ - Gabriele Poillucci ${ }^{2}$ Matthew D. Blackledge ${ }^{1,2}$. \\ David J. Collins ${ }^{1,2} \cdot$ Vallari Shah $^{3} \cdot$ Nina Tunariu ${ }^{1,2} \cdot$ Martin F. Kaiser ${ }^{3,4}$ • \\ Christina Messiou ${ }^{1,2}$
}

Received: 25 May 2017 /Revised: 31 August 2017 / Accepted: 13 September 2017 /Published online: 13 November 2017

(C) The Author(s) 2017. This article is an open access publication

\begin{abstract}
Objectives The aim of this study was to identify apparent diffusion coefficient (ADC) values for typical haemangiomas in the spine and to compare them with active malignant focal deposits. Methods This was a retrospective single-institution study. Whole-body magnetic resonance imaging (MRI) scans of 106 successive patients with active multiple myeloma, metastatic prostate or breast cancer were analysed. ADC values of typical vertebral haemangiomas and malignant focal deposits were recorded.

Results The ADC of haemangiomas (72 ROIs, median ADC $1,085 \times 10^{-6} \mathrm{~mm}^{2} \mathrm{~s}^{-1}$, interquartile range $927-1,295 \times 10^{-6} \mathrm{~mm}^{2} \mathrm{~s}^{-1}$ ) was significantly higher than the ADC of malignant focal deposits (97 ROIs, median ADC $682 \times 10^{-6} \mathrm{~mm}^{2} \mathrm{~s}^{-1}$, interquartile range $583-781 \times 10^{-6} \mathrm{~mm}^{2} \mathrm{~s}^{-1}$ ) with a $\mathrm{p}$-value $<10^{-6}$. Receiver operating characteristic (ROC) analysis produced an area under the curve of 0.93 . An ADC threshold of $872 \times 10^{-6} \mathrm{~mm}^{2} \mathrm{~s}^{-1}$ separated haemangiomas from malignant focal deposits with a sensitivity of $84.7 \%$ and specificity of $91.8 \%$.

Conclusions ADC values of classical vertebral haemangiomas are significantly higher than malignant focal deposits. The
\end{abstract}

Jessica M. Winfield

jessica.winfield@icr.ac.uk

1 Cancer Research UK Cancer Imaging Centre, Division of Radiotherapy and Imaging, The Institute of Cancer Research, 123 Old Brompton Road, London SW7 3RP, UK

2 Department of Radiology, MRI Unit, The Royal Marsden NHS Foundation Trust, Downs Road, Sutton, Surrey SM2 5PT, UK

3 Haemato-Oncology Research Unit, Division of Molecular Pathology, The Institute of Cancer Research, London, UK

4 Department of Haematology, The Royal Marsden NHS Foundation Trust, Downs Road, Sutton, Surrey SM2 5PT, UK high ADC of vertebral haemangiomas allows them to be distinguished visually and quantitatively from active sites of disease, which show restricted diffusion.

Key Points

- Whole-body diffusion-weighted MRI is becoming widely used in myeloma and bone metastases.

- $A D C$ values of vertebral haemangiomas are significantly higher than malignant focal deposits.

- High ADCs of haemangiomas allows them to be distinguished from active disease.

Keywords Diffusion magnetic resonance imaging - Whole body imaging $\cdot$ Bone neoplasms $\cdot$ Multiple myeloma .

Haemangioma

$\begin{array}{ll}\text { Abbreviations } \\ \text { ADC } & \text { Apparent diffusion coefficient } \\ \text { AP } & \text { Anterior-posterior } \\ \text { FOV } & \text { Field of view } \\ \text { GRAPPA } & \text { Generalized Autocalibrating Partially Parallel } \\ & \text { Acquisitions } \\ \text { MRI } & \text { Magnetic resonance imaging } \\ \text { PACS } & \text { Picture Archiving and Communication System } \\ \text { ROC } & \text { Receiver operating characteristic } \\ \text { ROI } & \text { Region of interest } \\ \text { STIR } & \text { Short-TI inversion recovery } \\ \mathrm{T}_{1} \mathrm{~W} & \mathrm{~T}_{1} \text { weighted } \\ \mathrm{T}_{2} \mathrm{~W} & \mathrm{~T}_{2} \text { weighted } \\ \mathrm{TE} & \text { Echo time } \\ \mathrm{TI} & \text { Inversion time } \\ \mathrm{TR} & \text { Repetition time }\end{array}$




\section{Introduction}

Vertebral haemangiomas are the most common benign vertebral neoplasm with a reported incidence of 10-26\% and are multiple in $7.2 \%$ of normal subjects [1]. They are most commonly asymptomatic, although rarely (0.9-1.2\% [2]) lesions can cause symptoms through local mass effect, fracture or bleeding [3]. Histologically vertebral haemangiomas are composed of newly formed blood vessels usually with normal structure in the absence of arteriovenous shunts. Haemangiomas are not separated from surrounding bone by a capsule and the surrounding osseous lamellae usually shows secondary osteolysis and osteocondensation, and the bone marrow undergoes fibrous and/or adipose involution [2].

The variable composition of vertebral haemangiomas does lead to some variation in MRI appearances but vertebral haemangiomas usually display classical MRI appearances. On $\mathrm{T}_{2}$-weighted $\left(\mathrm{T}_{2} \mathrm{w}\right)$ images typical haemangiomas return high-signal intensity due to slow flow in vascular channels and oedema. Fat content results in high signal on $\mathrm{T}_{1}$-weighted $\left(\mathrm{T}_{1} \mathrm{w}\right)$ imaging. The presence of high signal on $\mathrm{T}_{1} \mathrm{~W}$ or $\mathrm{T}_{2} \mathrm{~W}$ images is related to the amount of adipocytes or vessels and interstitial oedema, respectively $[4,5]$. Thickened trabeculae can sometimes be seen as linear low-signal intensity on all sequences [6]. As such, haemangiomas rarely present a diagnostic dilemma on routine MRI of the spine. However, applications for whole-body MRI are rapidly expanding and in particular whole-body MRI has become standard of care for myeloma and bone metastasis imaging in a growing number of institutions [7-9]. The complement of MR sequences used varies between institutions. For some applications such as screening for soft tissue malignancy in high-risk populations, whole-body MRI may consist of whole-body diffusionweighted MRI without $\mathrm{T}_{1} \mathrm{~W}$ and $\mathrm{T}_{2} \mathrm{~W}$ imaging of the spine. In the absence of standard MRI sequences, vertebral haemangiomas may therefore be mistaken for sinister focal deposits.

Furthermore, healthcare systems with limited resource will struggle to maximise the potential of whole-body diffusionweighted MRI. Automated segmentations made possible by machine learning technologies offer potential to not only speed up reporting times but also to transform whole-body qualitative data into quantitative datasets of disease burden and response to treatment [10]. Arguably the most exciting opportunities for such applications are in imaging bone disease but quantitative measures to enable exclusion of common vertebral haemangiomas from automated segmentations are not yet available.

In our institution whole-body diffusion-weighted MRI is standard of care for imaging patients with myeloma and is also frequently used to assess metastatic bone disease in patients with prostate or breast cancer. The potential mechanical complications in our patient population, which include vertebral fractures and spinal cord compression, necessitate inclusion of sagittal $\mathrm{T}_{1} \mathrm{~W}$ and $\mathrm{T}_{2} \mathrm{~W}$ MRI of the spine within the whole-body MRI protocol. This study therefore aims to identify apparent diffusion coefficient (ADC) values for typical haemangiomas in the spine and to compare them with malignant focal deposits.

\section{Materials and methods}

This was a retrospective single-institution study with local institutional review board approval.

\section{Subjects}

Whole-body MRI scans of 106 successive patients (57 prostate, 44 myeloma, five breast; 80 males, 26 females; median age 67 years, range $31-89$ years) with active multiple myeloma and focal deposits (as per International Myeloma Working Group criteria [11]) or metastatic bone disease from prostate and breast cancer (confirmed on $\mathrm{T}_{1} \mathrm{w}$ and $\mathrm{T}_{2} \mathrm{~W}$ MRI and sequential imaging) were included.

\section{MRI technique}

Using an Avanto $1.5 \mathrm{~T}$ system (Siemens, Erlangen, Germany) a whole-body study was achieved by the serial acquisition of contiguous body regions. All subjects were scanned supine with arms by their sides. Coil elements were positioned from skull vertex to knees. Sagittal $\mathrm{T}_{1} \mathrm{~W}$ images (TR $590 \mathrm{~ms}$, TE $11 \mathrm{~ms}$, field of view (FOV) 400 $\mathrm{mm}$, slice thickness $4 \mathrm{~mm}$ ), and $\mathrm{T}_{2} \mathrm{~W}$ images (TR 2,690 ms, TE $93 \mathrm{~ms}$, FOV $400 \mathrm{~mm}$, slice thickness $4 \mathrm{~mm}$ ) were acquired, followed by axial diffusion-weighted sequences (single-shot double spin echo echo-planar technique with STIR fat suppression in free breathing). b-values of 50 and $900 \mathrm{~s} \mathrm{~mm}^{-2}$ were applied in three orthogonal directions and combined to provide isotropic trace images. Diffusion-weighted images were acquired in multiple contiguous stations of 50 slices per station (slice thickness 5 $\mathrm{mm}$, no gap, FOV $430 \mathrm{~mm}$, phase direction AP, parallel imaging (GRAPPA) factor 2, TR $14,800 \mathrm{~ms}$, TE $66 \mathrm{~ms}$, inversion time (TI) $180 \mathrm{~ms}$, voxel size $2.9 \mathrm{~mm} \times 2.9 \mathrm{~mm}$ $\times 5 \mathrm{~mm}$, number of signal averages acquired 4, matrix $150 \times 150$, bandwidth $1,960 \mathrm{~Hz}$ per pixel). The scanner carrier frequency used for the most superior imaging station was applied for all other stations. The same shim gradient currents were applied for each station. Total acquisition time was $50 \mathrm{~min}$. 


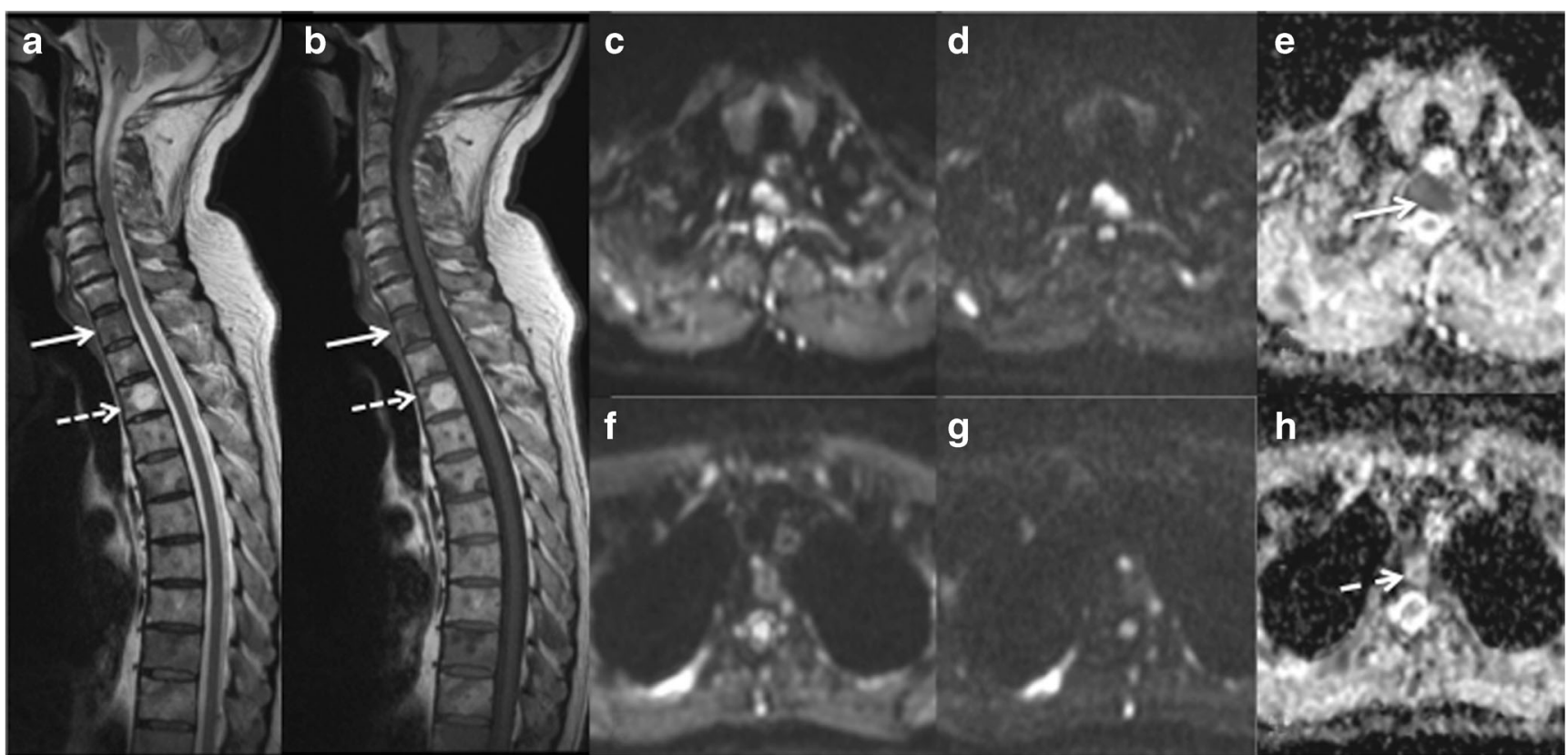

Fig. 1 (a) Sagittal $\mathrm{T}_{2} \mathrm{w}$ and (b) $\mathrm{T}_{1} \mathrm{w}$ MRI of the cervico-thoracic spine shows a typical low signal metastasis (arrow) and a typical high signal haemangioma (dashed arrow). Corresponding axial diffusion-weighted MRI images of the metastasis $\left(\mathbf{c} b=50 \mathrm{~s} \mathrm{~mm}^{-2}, \mathbf{d ~ b}=900 \mathrm{~s} \mathrm{~mm}^{-2}\right.$, and $\mathbf{e}$

\section{Image analysis}

For each patient a maximum of three vertebral haemangiomas measuring a minimum diameter of $1 \mathrm{~cm}$ were identified as bright focal lesions on sagittal $\mathrm{T}_{1} \mathrm{~W}$ and $\mathrm{T}_{2} \mathrm{~W}$ imaging of the spine (Fig. 1). With the aid of the Picture Archiving and Communication System (PACS) localiser the corresponding lesion was identified on the axial ADC map, which was generated using a monoexponential fit using the scanner's proprietary software. A region of interest (ROI) was drawn around the haemangioma and the mean ADC calculated for all pixels within the ROI. For each patient a maximum of three malignant focal deposits measuring a minimum diameter of $1 \mathrm{~cm}$ were identified on axial $b=900 \mathrm{~s}$ $\mathrm{mm}^{-2}$ diffusion-weighted images and ADC maps. An ROI was drawn around each deposit on the ADC map and the mean ADC calculated. The median diameter of ROIs was $18.6 \mathrm{~mm}$ (range $10.0-41.0 \mathrm{~mm})$.

\section{Statistical analysis}

Statistical analysis was carried out using Matlab version 2016a (The Mathworks, Natick, MA, USA). A two-sample t-test was used to assess whether there was a significant difference in ADC estimates between haemangiomas and malignant focal deposits. $\mathrm{P}<0.05$ was considered to indicate a significant difference. Receiver operating characteristic (ROC) curves were used to assess the performance of ADC estimates in distinguishing haemangiomas from malignant focal deposits. apparent diffusion coefficient (ADC) map) and of the haemangioma ( $\mathrm{b}=50 \mathrm{~s} \mathrm{~mm}^{-2}, \mathbf{g ~ b}=900 \mathrm{~s} \mathrm{~mm}^{-2}$, and $\mathbf{h}$ ADC map) are shown. The metastasis exhibits restricted diffusion on the ADC map (e, arrow) but conversely the haemangioma has a high $\mathrm{ADC}$ (h, dashed arrow)

\section{Results}

The ADC of haemangiomas ( 72 ROIs, median ADC $1,085 \times 10^{-}$ ${ }^{6} \mathrm{~mm}^{2} \mathrm{~s}^{-1}$, interquartile range $927-1,295 \times 10^{-6} \mathrm{~mm}^{2} \mathrm{~s}^{-1}$ ) was significantly higher than the ADC of malignant focal deposits $(97$ ROIs, median ADC $682 \times 10^{-6} \mathrm{~mm}^{2} \mathrm{~s}^{-1}$, interquartile range 583 $781 \times 10^{-6} \mathrm{~mm}^{2} \mathrm{~s}^{-1}$ ) with a p-value $<10^{-6}$ (Fig. 2).

ROC analysis produced an area under the curve of 0.93 . An ADC threshold of $872 \times 10^{-6} \mathrm{~mm}^{2} \mathrm{~s}^{-1}$ separated haemangiomas from malignant focal deposits with a sensitivity of $84.7 \%$ and specificity of $91.8 \%$ (Fig. 3).

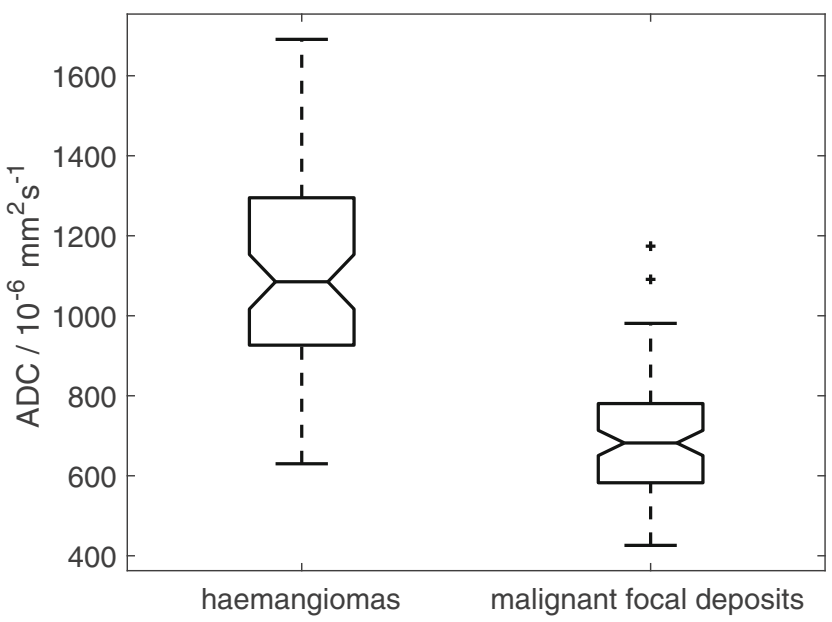

Fig. 2 Boxplot showing all apparent diffusion coefficient (ADC) estimates from haemangiomas $(n=72)$ and malignant focal deposits $(\mathrm{n}=97)$. Two-sample t-test, $\mathrm{p}<10^{-6}$ 


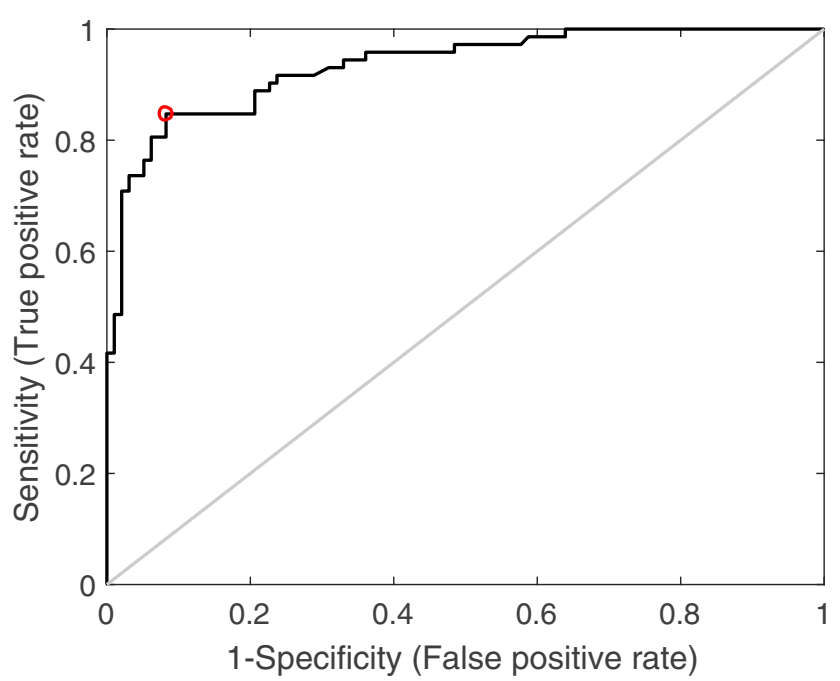

Fig. 3 Receiver operating characteristic (ROC) curve showing performance of apparent diffusion coefficient (ADC) estimates in distinguishing haemangiomas from malignant focal deposits. Red circle shows optimal operating point. Grey line shows line of identity

\section{Discussion}

This study demonstrated that ADC values of classical vertebral haemangiomas are significantly different to malignant focal deposits. The high ADC of vertebral haemangiomas allows them to be distinguished visually and quantitatively from active sites of disease, which show restricted diffusion. The ADC of malignant focal deposits $\left(682 \times 10^{-6} \mathrm{~mm}^{2} \mathrm{~s}^{-1}\right)$ was in agreement with previously published values of $761 \times 10^{-}$ ${ }^{6} \mathrm{~mm}^{2} \mathrm{~s}^{-1}$ for myeloma [12] and $782 \times 10^{-6} \mathrm{~mm}^{2} \mathrm{~s}^{-1}$ for bone metastases [13]. However, previous studies have reported ADC values of $960 \times 10^{-6} \mathrm{~mm}^{2} \mathrm{~s}^{-1}$ following treatment [12] and therefore treated sites of disease could potentially be mistaken for haemangiomas in the absence of corresponding $\mathrm{T}_{1} \mathrm{~W}$ and $\mathrm{T}_{2} \mathrm{~W}$ imaging or previous imaging for comparison.

The ADC values of vertebral haemangiomas and malignant focal deposits in the present study are higher than ADC values of normal bone marrow reported in previous studies of healthy volunteers, which were acquired with comparable protocols [14, 15]. Mean ADCs of (471 $\pm 142) \times 10^{-6} \mathrm{~mm}^{2} \mathrm{~s}^{-1}[14]$ and $(577.4 \pm 56.9) \times 10^{-6} \mathrm{~mm}^{2} \mathrm{~s}^{-1}$ [15] have been reported in bone marrow in mixed cohorts of healthy volunteers of comparable ages to the patients in the present study. We were unable to confirm normal marrow for analysis in this cohort of patients as bone marrow in patients with myeloma or bone metastases may be abnormal even in the absence of focal lesions or clear evidence of diffuse signal abnormality.

The narrow range of ADC estimates observed across the 97 malignant focal deposits suggests that the whole-body diffusion-weighted MRI protocol employed in this study is robust and suitable for use in quantitative applications. The wider range of ADC estimates observed across the 72 haemangiomas may arise from variations in the composition of haemangiomas and may relate to differences in vascularity, fat content and oedema.

Accurate estimation of ADCs across the FOV is essential for the use of $\mathrm{ADC}$ thresholds to discriminate between imaging features, for example to distinguish haemangiomas from malignant focal deposits. Variation in ADC estimates has been demonstrated at points far from isocentre and has been attributed largely to non-linearity in the diffusion-encoding gradients $[16,17]$. The variation in $\mathrm{ADC}$ estimates across the FOV in the whole-body diffusion-weighted MRI protocol employed in this study has been shown to be around 5-8\% but greater variations in ADC estimates would be encountered in imaging protocols that employ larger numbers of slices per station [17].

In conclusion, the ADC values of classical vertebral haemangiomas are significantly higher than the ADCs of malignant focal deposits. The high ADC of vertebral haemangiomas allows them to be distinguished visually and quantitatively from active sites of disease in whole-body diffusion-weighted imaging.

Acknowledgements This report is independent research funded by the National Institute for Health Research. The views expressed in this publication are those of the author(s) and not necessarily those of the National Health Service, the National Institute for Health Research or the Department of Health.

Funding This study has received funding by Cancer Research UK and Engineering and Physical Sciences Research Council support to the Cancer Imaging Centre at The Institute of Cancer Research and Royal Marsden Hospital in association with Medical Research Council and Department of Health C1060/A10334, C1060/A16464 and National Health Service funding to the National Institute for Health Research Biomedical Research Centre, Clinical Research Facility in Imaging and the Cancer Research Network.

\section{Compliance with ethical standards}

Guarantor The scientific guarantor of this publication is Dr Christina Messiou.

Conflict of interest The authors of this manuscript declare no relationships with any companies whose products or services may be related to the subject matter of the article.

Statistics and biometry No complex statistical methods were necessary for this paper.

Informed consent Written informed consent was waived by the Institutional Review Board.

Ethical approval Institutional Review Board approval was obtained.

\author{
Methodology \\ - retrospective \\ - observational \\ - performed at one institution
}


Open Access This article is distributed under the terms of the Creative Commons Attribution 4.0 International License (http:// creativecommons.org/licenses/by/4.0/), which permits unrestricted use, distribution, and reproduction in any medium, provided you give appropriate credit to the original author(s) and the source, provide a link to the Creative Commons license, and indicate if changes were made.

\section{References}

1. Slon V, Stein D, Cohen H, Sella-Tunis T, May H, Hershkovitz I (2015) Vertebral hemangiomas: their demographical characteristics, location along the spine and position within the vertebral body. Eur Spine J 24:2189-2195

2. Pastushyn AI, Slin'ko EI, Mirzoyeva GM (1998) Vertebral hemangiomas: diagnosis, management, natural history and clinicopathological correlates in 86 patients. Surg Neurol 50:535-547

3. Vinay S, Khan SK, Braybrooke JR (2011) Lumbar vertebral haemangioma causing pathological fracture, epidural haemorrhage, and cord compression: a case report and review of literature. J Spinal Cord Med 34:335-339

4. Baudrez V, Galant C, Vande Berg BC (2001) Benign vertebral hemangioma: MR-histological correlation. Skeletal Radiol 30: 442-446

5. Ross JS, Masaryk TJ, Modic MT, Carter JR, Mapstone T, Dengel FH (1987) Vertebral hemangiomas: MR imaging. Radiology 165: 165-169

6. Nouh MR, Eid AF (2015) Magnetic resonance imaging of the spinal marrow: basic understanding of the normal marrow pattern and its variant. World J Radiol 7:448-458

7. National Institute for Health and Care Excellence (2016) Myeloma: Diagnosis and Management. NICE guideline (NG35). Available via https://www.nice.org.uk/guidance/ng35/chapter/recommendations. Accessed 24 May 2017
8. Messiou C, Kaiser M (2015) Whole body diffusion weighted MRI a new view of myeloma. Br J Haematol 171:29-37

9. Padhani AR, Lecouvert FE, Tunariu N et al (2017) METastasis reporting and data system for prostate cancer: practical guidelines for acquisition, interpretation, and reporting of whole-body magnetic resonance imaging-based evaluations of multiorgan involvement in advanced prostate cancer. Eur Urol 71:81-92

10. Rueckert D, Glocker B, Kainz B (2016) Learning clinically useful information from images: Past, present and future. Med Image Anal 33:13-18

11. Dimopoulos MA, Hillengass J, Usmani S et al (2015) Role of magnetic resonance imaging in the management of patients with multiple myeloma: a consensus statement. J Clin Oncol 33:657-664

12. Messiou C, Giles S, Collins DJ et al (2012) Assessing response of myeloma bone disease with diffusion-weighted MRI. Br J Radiol 85:e1198-e1203

13. Perez-Lopez R, Mateo J, Mossop H et al (2017) Diffusion-weighted imaging as a treatment response biomarker for evaluating bone metastases in prostate cancer: a pilot study. Radiology 283:168-177

14. Messiou C, Collins DJ, Morgan VA, deSouza NM (2011) Optimising diffusion weighted MRI for imaging metastatic and myeloma bone disease and assessing reproducibility. Eur Radiol 21:1713-1718

15. Lavdas I, Rockall AG, Castelli F et al (2015) Apparent diffusion coefficient of normal abdominal organs and bone marrow from whole-body DWI at 1.5T: the effect of sex and age. AJR AM J Roentgenol 205:242-250

16. Malyarenko DI, Newitt D, Wilmes LJ et al (2016) Demonstration of nonlinearity bias in the measurement of the apparent diffusion coefficient in multicenter trials. Magn Reson Med 75:1312-1323

17. Winfield JM, Collins DJ, Priest AN et al (2016) A framework for optimization of diffusion-weighted MRI protocols for large fieldof-view abdominal-pelvic imaging in multicenter studies. Med Phys 43:95-110 